\title{
The expression of BRCA1, P53, KAI1, and Nm23 in ovaries of BRCA1 mutation carriers after prophylactic adnexectomy
}

\author{
J. Markowska $\cdot$ J. Bar $\cdot$ R. Mądry $\cdot$ \\ I. Słomska $\cdot$ M. Mardas $\cdot$ J. P. Grabowski
}

Received: 19 March 2012/Accepted: 22 March 2013/Published online: 4 April 2013

(C) The Author(s) 2013. This article is published with open access at Springerlink.com

\begin{abstract}
Introduction High mortality rate, absence of reliable methods for early diagnosis and poor prognosis of advanced ovarian cancer prompted to investigate the role of prophylactic oophorectomy in BRCA1 mutation carriers as well as evaluate the expression of BRCA1, p53, Nm23, and KAI1 proteins in ovarian tissue from these patients.

Materials and methods Ovaries from BRCA1 mutation carriers underwent prophylactic adnexectomy and control group of patients were operated from other than cancer reasons. The expression of selected proteins was studied using immunohistochemical staining. The intensity of immunostaining and the number of tumor cells showing the reaction for selected proteins were analyzed.

Results We have analyzed ovarian tissues from 18 BRCA1 mutation carriers and 11 women included in control group. Positive expression of BRCA1 protein was presented in $83.3 \%$ cases in BRCA1 mutation carriers and in $72.7 \%$ in the control group $(p>0.05)$. Positive expression of $\mathrm{p} 53$ protein was observed, respectively, in 27.8 vs. $36.4 \%(p>0.05), \mathrm{Nm} 23$ protein 77.7 vs. $90.9 \%$ $(p>0.05)$, and KAI1 in 72.2 vs. $72.7 \%(p>0.05)$. Mean percent of tumor cells that showed the reaction for selected
\end{abstract}

J. Markowska · R. Mądry · M. Mardas · J. P. Grabowski Division of Gynecology, Department of Oncology, Poznan University of Medical Sciences, Poznan, Poland

J. Bar · I. Słomska

Department of Clinical Immunology, Wrocław Medical

University, Wrocław, Poland

J. P. Grabowski $(\bowtie)$

Department of Gynecology and Gynecological Oncology, Kliniken-Essen-Mitte, Henricistr. 92, 45136 Essen, Germany e-mail: jacgrab@yahoo.com proteins as well as the intensity of immunostaining for all analyzed proteins seems to be lower in BRCA1 mutation carriers.

Conclusions However, any significant differences between study group and control group have not been found; there were similar trends showing reduced expression of studied proteins in BRCA1 mutation carriers.

Keywords BRCA1 - Prophylactic adnexectomy

\section{Introduction}

Epithelial ovarian cancer is often referred as the "silent killer" according to its asymptomatic clinical pattern, mostly diagnosed in its advanced stages [1]. Approximately $10 \%$ of ovarian cancer cases are attributed to the inheritance of a BRCA1 or 2 mutation [2]. The lifetime risk of ovarian cancer in patients with a BRCA1 mutation is between 40 and $60 \%[1,3]$ compared with the general population risk of $1.8 \%$ [4]. Due to high mortality rate, absence of reliable methods for early diagnosis and bad prognosis of advanced stages of ovarian cancer the role of prophylactic adnexectomy remain the therapy of choice [2, 4]. Especially, assuming significant lifetime risk reduction for developing ovarian cancer and a low probability of peritoneal dissemination according to three cohort studies in patients undergoing bilateral adnexectomy [2].

BRCA1 was identified and cloned in 1994 [1]. This gene is localized on chromosome 17 (17q21) and codes large proteins that consist of 1,863 amino acids. BRCA1 protein has a large number of functions, including regulation of transcription, embryological development, immune modulation, cell cycle control, and DNA repair [5, 6]. The loss of the last function leads to accumulation of unrepaired 
somatic mutations what may result in development of ovarian cancer [5].

The p53 protein has an important biologic function because it interferes in the response to DNA damage. The protein accumulates in the cell nucleus and is activated as a transcription factor in response to DNA damage, hypoxia, and other genotoxic stresses. It can block the cell cycle in $\mathrm{G} 1$, allowing the cell to repair genomic damage, or it can induce apoptosis. Loss of p53 function plays a central role in the development of cancer. In fact, p53 is mutated in $40-80 \%$ of epithelial ovarian cancers [6, 7]. It is known that BRCA1 interacts with p53 in the normal tumor suppressor pathway. A higher prevalence of p53 mutations has been identified in the BRCA related tumors than in sporadic ovarian cancer [5].

A metastasis suppressor gene on human chromosome 11 p11.2 that encodes a glycoprotein of the transmembrane four superfamily (TM4SF) is the highly glycosylated protein KAI1 (CD 82). Although the precise biological functions of TM4SF molecules are not fully elucidated, several studies have shown involvement in cell growth, adhesion, and motility. This protein is physiologically expressed in a variety of tissues and was reported to be downregulated in several types of human cancer, including cancer of the ovary, cervix, prostate, lung, breast, bladder, colon, and pancreas [8-10].

Nm23 gene product has activity of nucleoside diphosphate kinase (NDPK). Gene has been reported to be involved in cell proliferation and differentiation. Reduced expression of the $\mathrm{Nm} 23$ gene is implicated in the metastatic progression of various cancers: breast, ovary, cervix, liver, colon, gastric, and melanoma. Although expression of $\mathrm{Nm} 23$ is divergent in various malignant tumors, its downregulated expression seems to be related with increased metastatic potential in most carcinoma types [11-13].

The aim of this study was to investigate the expression of BRCA1 protein as well as p53, Nm23, and KAI1 proteins associated with tumor metastatic progression in ovarian tissue removed during prophylactic adnexectomy in BRCA1 mutation carriers.

\section{Materials and methods}

Ovarian tissue was collected from BRCA1 mutation positive patients during prophylactic bilateral adnexectomy. As a control group, samples from patients operated in our Department from other than cancer reason were evaluated. All patients in control group were not BRCA mutation carriers.

Immunohistochemical staining for analyzed proteins was performed on frozen 5 - $\mu$ m tissue sections using the
Universal DakoCytomation LSAB + Kit, Peroxidase procedure (LSAB + Kit: HRP, Dako, Copenhagen, Denmark) and following primary antibodies: monoclonal antibody anti-p53 protein (clone DO-7) (Novocastra, Newcastle, UK), monoclonal antibody anti-nm23 protein (Novocastra), monoclonal antibody anti-KAI 1 (G-2) (Santa Cruz, USA), and polyclonal antibody anti-BRCA1 (C-20) protein (Santa Cruz). Nonspecific tissue and endogenous peroxidase reactivity were blocked with $10 \%$ BSA (Bovine serum albumin) and $3 \% \mathrm{H}_{2} \mathrm{O}_{2}$, respectively. Tissue specimens were incubated with primary antibodies $60 \mathrm{~min}$ at room temperature. Following washing with $0.1 \mathrm{M}$ Trisbuffer, pH 7.4 (TBS), the tissue specimens were incubated with secondary biotinylated rabbit antibody, anti-mouse/ rabbit IgG (Dako, Denmark) and with streptavidin horseradish peroxidase conjugated (Dako) both for $15 \mathrm{~min}$ at room temperature. After washing with TBS, the antigenantibody reaction was visualized by DAB (3,3 diaminobenzidine) (Dako, Denmark) as a chromogen (8 min, room temperature). Sections were counterstained with hematoxylin and mounted. The incubation buffer (TBS) without primary antibodies was used as negative control. The internal positive controls were used.

The evaluation of immunohistochemical data was performed as following. Sections were scored semiquantitatively, taking into account the intensity of immunostaining and the number of tumor cells showing the reaction for p53, Nm23, KAI1, and BRCA1 protein. The cases were score as positive for p53 protein expression when, more than $15 \%$ of the tumor cells revealed nuclear immunopositivity. KAI1, Nm23, and BRCA1 expression was assessed positive if more than $10 \%$ of tissue section showed immunostaining.

Statistical analysis has been performed using STATISTICA 6.0.

\section{Results}

Overall, ovarian tissues from 29 patients were examined. Studied group included $18(62 \%)$ patients with BRCA1 mutation (mean age $50.1 \pm 8.2$ ). Among those, 10 women had previous history of breast cancer. Control group included 11 (38\%) patients operated from other than cancer reasons (mean age $51.6 \pm 10.2$ ). We did not observe any postoperative complications.

In one woman from a study group, high-grade serous ovarian cancer was diagnosed. This patient has not suffered from a breast cancer in the past. After completing staging surgery, tumor was classified as FIGO IC.

As shown in Fig. 1 expression of BRCA1 protein in ovarian tissue was presented in $83.3 \%$ (15 pat.) BRCA1 mutation carriers and in $72.7 \%$ (8 pat.) patients in control 


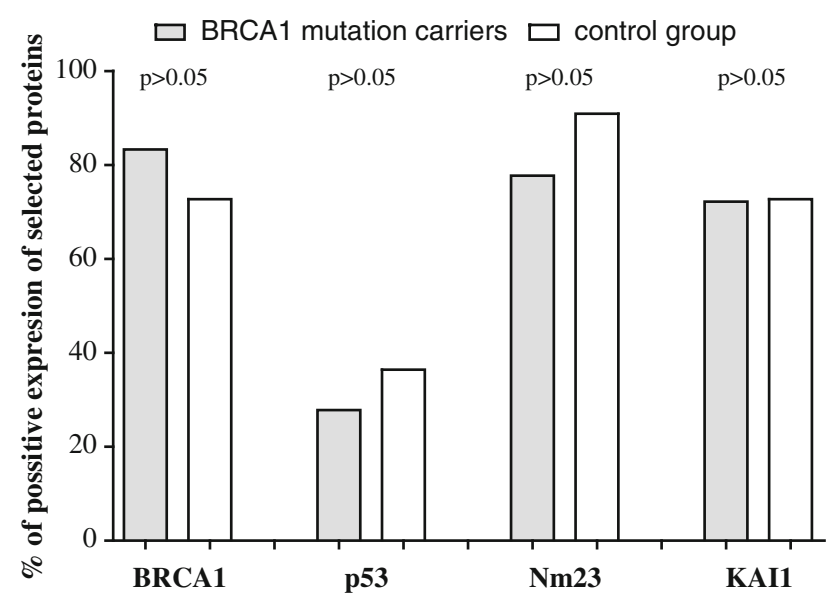

Fig. 1 The percent of positive expression of BRCA1, p53, Nm23, and KAI1 proteins in BRCA1 mutation carriers and control group

group. Positive expression of p53 protein was observed, respectively, in $27.8 \%$ (5 pat.) vs. $36.4 \%$ (4 pat.), of Nm23 protein $77.7 \%$ (14 pat.) vs. $90.9 \%$ (10 pat.), and of KAI1 in $72.2 \%$ (13 pat.) vs. $72.7 \%$ (8 pat.) cases. Mean percentage values of tumor cells showing the reaction for BRCA1, p53, Nm23, and KAI1 proteins are presented in Fig. 2. It has been identified that mean cell percentage in which expression of BRCA1 protein was identified in BRCA1 mutation carriers which reached $33.3 \%$ (6 pat.) and in the control group $45.4 \%$ (5 pat.), respectively. p53 protein was detected in $5.6 \%$ (1 pat.) vs. $18.1 \%$ (2 pat.), Nm23 protein $22.2 \%$ (4 pat.) vs. $27.3 \%$ (3 pat.), and KAI1 $33.3 \%$ (6 pat.) vs. $45.4 \%$ (5 pat.) of cells, respectively. Mean values of the intensity of immunostaining for all analyzed proteins are presented in Fig. 3. For BRCA1 protein, mean value of intensity of immunostaining in BRCA1 mutation positive group was $1.26 \mathrm{ou} / \mu^{2}$ and in

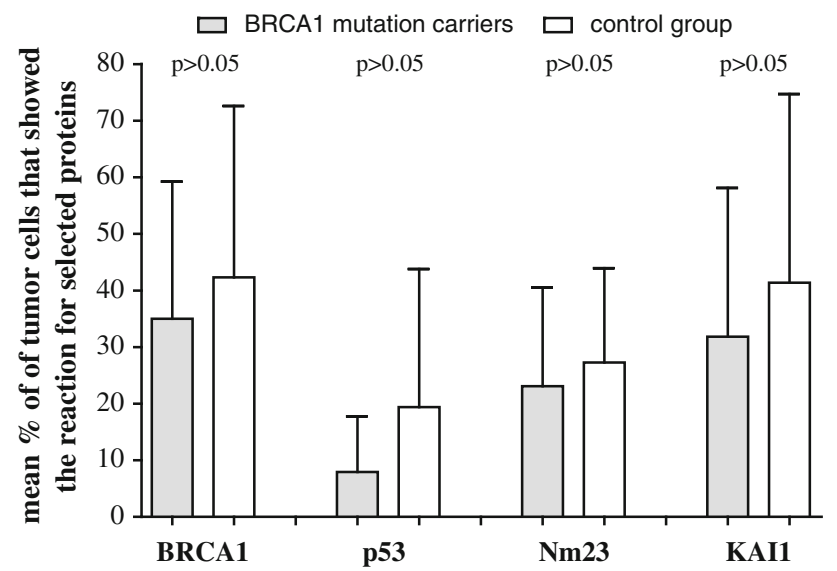

Fig. 2 Mean percentage value of the tumor cells showing the reaction for BRCA1, p53, Nm23, and KAI1 proteins

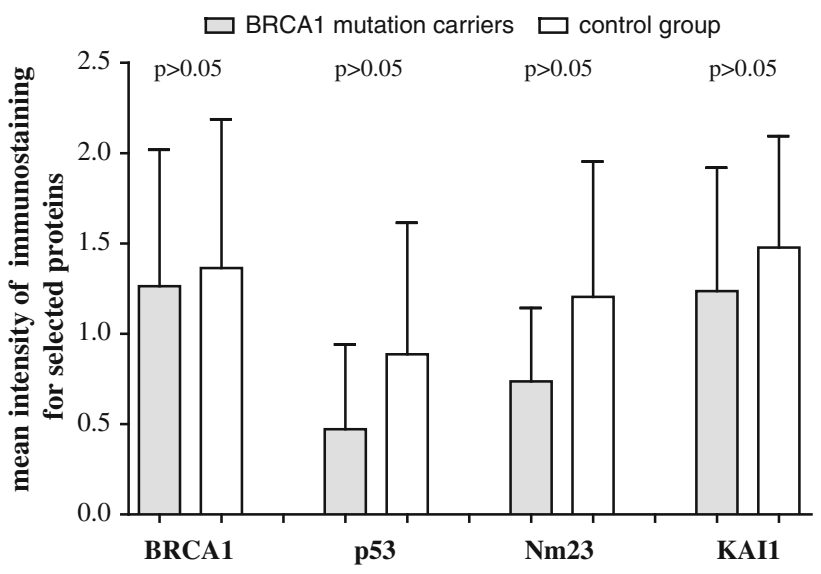

Fig. 3 Mean values of the intensity of immunostaining $\left(\mathrm{ou} / \mu \mathrm{m}^{2}\right)$ for BRCA 1, p53, Nm23, and KAI1 proteins

the control group $1.36 \mathrm{ou} / \mu^{2}$. Accordingly, for p53 protein 0.47 vs. $0.88 \mathrm{ou} / \mu \mathrm{m}^{2}$, for $\mathrm{Nm} 23$ protein 0.73 vs. 1.20 $\mathrm{ou} / \mu \mathrm{m}^{2}$, and for KAI1 1.24 vs. $1.47 \mathrm{ou} / \mu \mathrm{m}^{2}$.

The group of BRCA1 mutation carriers has been additionally evaluated according to medical history of breast cancer. Positive expression of BRCA1 protein was present in nine patients $(90.0 \%)$ in the group with breast cancer and in six patients $(75.0 \%)$ in BRCA1 mutation carriers without breast cancer. Positive expression of $\mathrm{p} 53$ protein was observed, respectively, in 4 cases $(40.0 \%)$ vs. 1 case $(12.5 \%)$, of $\mathrm{Nm} 23$ protein $8(80 . \%)$ vs. $6(75.0 \%)$, and of KAI1 in $7(70 . \%)$ vs. $6(75.0 \%)$. Mean percentage values of cells showing the reaction for BRCA1 were $30.0 \%$ in breast cancer group vs. $37.5 \%$ in BRCA1 mutation carriers without breast cancer. Mean percentage values of the tumor cells showing the reaction for p53 protein were, respectively, 0 vs. $12,5 \%$, for $\mathrm{Nm} 2320.0$ vs. $25.0 \%$, and for KAI1 30.0 vs. $37.5 \%$. Mean values of the intensity of immunostaining for BRCA1 protein were $1.0 \mathrm{ou} / \mu \mathrm{m}^{2}$ in the group with breast cancer versus $1.3 \mathrm{ou} / \mu \mathrm{m}^{2}$ in BRCA1 mutation carriers only without breast cancer. Mean values of the intensity of immunostaining for $\mathrm{p} 53$ protein were, respectively, 0.41 vs. $0.53 \mathrm{ou} / \mu^{2}$, for $\mathrm{Nm} 230.72$ vs. 0.75 $\mathrm{ou} / \mu \mathrm{m}^{2}$, and for KAI1 $1.1 \mathrm{vs} .1 .4 \mathrm{ou} / \mu \mathrm{m}^{2}$.

Three types of BRCA1 mutations were identified within the patients in our study. We have not found differences in patients showing expression of BRCA1, p53, KAI1, and $\mathrm{Nm} 23$ proteins regarding the type of mutation and breast cancer history (Table 1). According to percent of cells showing expression of studied genes, we have observed lower values in patients with breast cancer history in all types of BRCA1 mutations (Table 2). In Table 3 are presented mean values of the intensity of immunostaining that did not present significant differences. 
Table 1 BRCA1 mutations carriers showing expression of BRCA1, p53, KAI1, and Nm23 proteins

\begin{tabular}{lllll}
\hline BRCA1 mutation type & BRCA1 & p53 & KAI1 & Nm23 \\
\hline ex 20 $(5382$ ins C) & & & \\
$\quad$ All $(n=9)$ & $8(88.9 \%)$ & $3(33.3 \%)$ & $6(66.7 \%)$ & $7(77.8 \%)$ \\
BC $(n=6)$ & $6(100 \%)$ & $2(33.3 \%)$ & $4(66.7 \%)$ & $4(66.7 \%)$ \\
ex $5(300 \mathrm{~T} / \mathrm{G})$ & & & \\
All $(n=7)$ & $5(71.4 \%)$ & $1(14.3 \%)$ & $5(71.4 \%)$ & $5(71.4 \%)$ \\
BC $(n=3)$ & $2(66.7 \%)$ & $0(0 \%)$ & $3(100 \%)$ & $3(100 \%)$ \\
ex $11.17(4153$ del A) & & & & \\
All $(n=2)$ & $2(100 \%)$ & $1(50 \%)$ & $2(100 \%)$ & $2(100 \%)$ \\
BC $(n=1)$ & $1(100 \%)$ & $1(100 \%)$ & $1(100 \%)$ & $1(100 \%)$ \\
\hline
\end{tabular}

Table 2 Mean percentage value of cells showing the reaction for BRCA1, p53, KAI1, and Nm23 in BRCA1 mutation carriers indicating the type of BRCA1 mutation and the breast cancer history

\begin{tabular}{lllll}
\hline $\begin{array}{l}\text { BRCA1 mutation } \\
\text { type }\end{array}$ & $\begin{array}{l}\text { BRCA1 } \\
(\%)\end{array}$ & $\begin{array}{l}\text { p53 } \\
(\%)\end{array}$ & $\begin{array}{l}\text { KAI1 } \\
(\%)\end{array}$ & $\begin{array}{l}\text { Nm23 } \\
(\%)\end{array}$ \\
\hline ex 20 (5382 ins C) & & & & \\
$\quad$ No BC $(n=3)$ & 38.3 & 8.9 & 40.8 & 25.1 \\
BC $(n=6)$ & 47 & 6.7 & 38.3 & 21.8 \\
ex 5 (300 T/G) & & & & \\
No BC $(n=4)$ & 36.3 & 7.5 & 46.7 & 7.5 \\
BC $(n=3)$ & 33.3 & 3.3 & 18.8 & 31.7 \\
ex 11.17 $(4153$ del A) & & & & \\
No BC $(n=1)$ & 30 & 11.4 & 47.5 & 32.5 \\
BC $(n=1)$ & 20 & 15 & 15 & 25 \\
\hline
\end{tabular}

$B C$ breast cancer history, $n$ number of patients

Table 3 Mean values of the intensity of immunostaining $\left(\mathrm{ou} / \mu \mathrm{m}^{2}\right)$ for BRCA1, p53, KAI1, and Nm23 in BRCA1 mutation carriers indicating the type of BRCA1 mutation and the breast cancer history

\begin{tabular}{lllll}
\hline BRCA1 mutation type & BRCA1 & p53 & KAI1 & Nm23 \\
\hline ex 20 (5382 ins C) & & & & \\
No BC $(n=3)$ & 0.67 & 0.58 & 1.5 & 1 \\
BC $(n=6)$ & 1.38 & 0.42 & 1.21 & 0.63 \\
ex 5 (300 T/G) & & & & \\
No BC $(n=4)$ & 1.31 & 0.31 & 0.75 & 0.56 \\
BC $(n=3)$ & 1 & 0.33 & 1.58 & 0.75 \\
ex 11.17 (4153 del A) & & & & \\
No BC $(n=1)$ & 1.75 & 1 & 1.75 & 0.75 \\
BC $(n=1)$ & 2.5 & 1 & 1.75 & 1.25 \\
\hline
\end{tabular}

$B C$ breast cancer history, $n$ number of patients

\section{Discussion}

Recent progress in our understanding of familiar ovarian cancer has led to significant changes in the day-to-day practice of surgical pathology [5]. Three cohort studies have identified a risk reduction with prophylactic bilateral adnexectomy in women with germ line BRCA1 or 2 mutations by comparing the incidence of ovarian cancer in the control group to the incidence of primary peritoneal carcinoma in the prophylactic adnexectomy group. Assumed analysis showed significant lifetime risk reduction for developing ovarian cancer and a low probability of peritoneal cancer in those undergoing surgery [2]. The development of cancer in BRCA germline mutation carriers occurs only if there is subsequent inactivation of the remaining wild-type BRCA allele on the opposite chromosome in addition to the pre-existing BRCA germline mutation [5].

In the study of Wang et al. [14], decreased expression of BRCA1 was found in $16 \%$ of benign tumors, $38 \%$ of borderline tumors, and $72 \%$ of carcinomas. These results suggest that downregulation of BRCA1 protein play an important role in the development ovarian cancers [14]. In our study, positive expression of BRCA1 protein was observed $83.3 \%$ of BRCA1 mutation carriers in comparison to $72.7 \%$ in control group; however, the mean percentage value of the tumor cells showing the reaction for BRCA1 protein in BRCA1 mutation carriers was reduced in comparison to control group. The same results were observed in the intensity of immunostaining. Patients with history of breast cancer have higher expression rate of BRCA1; however, regarding to mutation of this gene function its product is impaired.

Loss of p53 function plays a central role in the development of cancer. The biological consequence of a missense mutation is enhancement of p53 stability and accumulation in the tumor cell nucleus [7]. The p53 alterations indisputably occur more often in BRCA1associated tumors than in sporadic breast or ovarian tumors. This implies that loss of p53 function is a critical event in the molecular pathogenesis of BRCA1-associated tumors [15]. In ovarian cancer, immunohistochemical detectable overexpression of p53 is highly associated with presence of mutated, nonfunctional p53 [16]. However, Canevari et al. [7] suggest that p53 mutation is a late event in ovary carcinogenesis. In our study, the expression, mean 
percentage value of tumor cells presenting expression as well as intensity of immunostaining of p53 in BRCA1 mutation carriers were reduced in comparison to the control group. Lowest values have been noted in the study group patients with a history of breast cancer.

KAI1 is well known as a prostate cancer gene [9]. A report by Liu et al. [17] suggested that the downregulation of KAI1 expression may have a negative impact on survival in ovarian cancer. Also results by Houle et al. [9] suggest that the malignant progression of epithelial ovarian carcinomas is associated with downregulation and altered cellular localization of KAI1. Liu et al. [17] were unable to find any mutation of the KAI1 gene in primary or recurrent ovarian carcinomas except a missense polymorphism in codon 241. This finding confirms the observation that downregulation, rather than mutation, is a more common mechanism for the dysregulation of the KAI1 gene [17]. In human prostatic cancer, the expression of KAI1 was reported to be strongly correlated with that of p53, and the loss of both proteins was associated with poor survival [18]. This correlation was not found in ovarian carcinoma [8]. Houle et al. [9] observed a shift in protein localization of KAI1 from the membrane in grade 1 tumors to the cytoplasm in grade 3 tumors. They suggest that these changing patterns of expression from the membrane may be a mechanism by which tumor cells lose their adhesive properties during malignant transformation. In our study, expression of KAI1 in samples from BRCA1 mutation carriers seems to be downregulated again with lowest values in patients with a history of previous breast cancer. Also in our study, no correlation between KAI1 and p53 was found.

As the Nm23/NDP kinase-A gene was discovered in a murine melanoma metastasis model system, the correlation of its expression with tumor metastatic potential in actual human cancers was a subject of great interest [19]. However, breast carcinoma and melanoma are the two tumor types most studied in this context [19]; there are also reports in ovarian carcinoma $[12,20]$. In study by Gao et al. [20], the expression of Nm23 RNA in human ovarian cancer cells was inversely related to metastatic behavior in the experimental animals and strongly suggested that the $\mathrm{Nm} 23$ expression correlates with reduced metastasis of ovarian carcinoma. Elevated $\mathrm{Nm} 23$ expression is also related to lower rates of lymph node metastasis and longer survival in endometrial cancer. Lower expression of $\mathrm{Nm} 23$ is an indicator of poor prognosis and may increase the risk of regional lymph node metastasis [21]. Adverse results reported by Srivatsa et al. [12] showed that expression of $\mathrm{Nm} 23$ is strongly upregulated in most epithelial ovarian cancer and suggest that $\mathrm{Nm} 23$ gene expression may have distinct if not opposite biologic functions in epithelial ovarian cancer and breast carcinoma.

\section{Conclusions}

Observed lower expression of BRCA1, KAI1, NM23, and p53 may suggest the role of these proteins in carcinogenesis and poorer prognosis of hereditary ovarian cancer. However, no significant statistical differences between relatively small studied groups were found, the trends suggesting downregulation of these proteins in BRCA1 mutation carriers was reported. Lowest values were observed in patients with previous history of breast cancer of study group what might suggest that downregulation of studied proteins is combined with higher risk of carcinogenesis.

Conflict of interest No potential conflict of interest relevant to this article was reported.

Open Access This article is distributed under the terms of the Creative Commons Attribution License which permits any use, distribution, and reproduction in any medium, provided the original author(s) and the source are credited.

\section{References}

1. Reynolds EA, Moller KA (2006) A review and an update on the screening of epithelial ovarian cancer. Curr Probl Cancer 30:203-232

2. Rosen B, Kwon J, Fung Kee Fung M, Gagliardi A, Chambers A (2004) Systematic review of management options for women with a hereditary predisposition to ovarian cancer. Gynecol Oncol 93:280-286

3. Seeber B, Driscoll DA (2004) Hereditary breast and ovarian cancer syndrome: should we test adolescents? J Pediatr Adolesc Gynecol 17:161-167

4. Palma M, Ristori E, Ricevuto E, Giannini G, Gulino A (2006) BRCA1 and BRCA2: the genetic testing and the current management options for mutation carriers. Criti Rev Oncol Hemat 57:1-23

5. Weinreb I, Colgan TJ (2004) BRCA mutations and pathology in the female genital tract. Curr Diagn Pathol 10:326-335

6. Nicoletto MO, Donach M, De Nicolo A, Artioli G, Banna G, Monfardini S (2001) BRCA-1 and BRCA-2 mutations as prognostic factors in clinical practice and genetic counselling. Cancer Treat Rev 27:295-304

7. Canevari S, Gariboldi M, Reid JF, Bongarzone I, Pierotti MA (2006) Molecular predictors of response and outcome in ovarian cancer. Criti Rev Oncol Hemat 60:19-37

8. Schindl M, Birner P, Breitenecker G, Oberhuber G (2001) Downregulation of KAI1 metastasis suppressor protein is associated with a dismal prognosis in epithelial ovarian cancer. Gynecol Oncol 83:244-248

9. Houle CD, Ding XY, Foley JF, Afshari CA, Barrett JC, Davis BJ (2002) Loss of expression and altered localization of KAI1 and CD9 protein are associated with epithelial ovarian cancer progression. Gynecol Oncol 86:69-78

10. White A, Lamb PW, Barret JC (1998) Frequent downregulation of the KAI1(CD82) metastasis suppressor protein in human cancer cell lines. Oncogene 16:3143-3149

11. Cipollini G, Moretti A, Ghimenti C, Viacava P, Bevilacqua G, Caligo MA (2000) Mutational analysis of the NM23.H1 gene in human breast cancer. Cancer Genet Cytogenet 121:181-185 
12. Srivatsa PJ, Cliby WA, Keeney GL, Dodson MK, Suman VJ, Roche PC, Podratz KC (1996) Elevated nm23 protein expression is correlated with diminished progression-free survival in patients with epithelial ovarian carcinoma. Gynecol Oncol 60:363-372

13. Tee YT, Chen GD, Lin LY, Ko JL, Wang PH (2006) Nm23-h1: a metastasis-associated gene. Taiwanese J Obstet Gynecol 45(2):107-113

14. Wang C, Horiuchi A, Imai T, Ohira S, Itoh K, Nikaido T et al (2004) Expression of BRCA1 protein in benign, borderline, and malignant epithelial ovarian neoplasms and its relationship to methylation and allelic loss of the BRCA1 gene. J Pathol 202:215-223

15. Schuyer M, Berns EMJJ (1999) Is TP53 dysfunction required for BRCA1-associated carcinogenesis? Mol Cel Endocrinol 155:143-152

16. DiCioccio RA, Werness BA, Peng R, Allen HJ, Piver MS (1998) Correlation of TP53 mutations and p53 overexpression in ovarian tumors. Cancer Genet Cytogenet 105:93-102
17. Liu FS, Dong JT, Chen JT, Hsieh YT, Ho ES, Hung MJ (2000) Frequent down-regulation and lack of mutation of the KAI1 metastasis suppressor in epithelial ovarian carcinoma. Gynecol Oncol 78:10-15

18. Mashimo T, Watabe M, Hirota S, Hosobe S, Miura K, Tegtmeyer PJ, Rinker-Shaeffer CW, Watabe K (1998) The expression of the KAI1 gene, a tumor metastasis suppressor, is directly acivated by p53. Proc Natl Acad Sci USA 95:11307-11311

19. Hartsough MT, Steeg PS (2000) Nm23/Nucleoside diphosphate kinase in human cancers. J Bioenerg Biomemb 32(3):301-308

20. Gao Q, Ma D, Meng L, Wang S, Wang C, Lu Y et al (2004) Related Study on the Nm23-H1 Gene Expression in the Model of Ovarian Carcinoma Cell Lines with High Frequent Metastasis. Chin-Ger J Clinic Oncol 3(2):97-100

21. Yalcinkaya U, Özuysal S, Bilgin T, Ercan I, Saraydaroglu Ö, Demir D (2006) Nm23 expression in node-positive and nodenegative endometrial cancer. Int J Gyn Obstet 95:35-39 